\title{
Comparison of two techniques for reliable characterization of thin metal-dielectric films
}

\author{
Tatiana V. Amotchkina, ${ }^{1, *}$ Michael K. Trubetskov, ${ }^{1}$ Alexander V. Tikhonravov, ${ }^{1}$ \\ Vesna Janicki, ${ }^{2}$ Jordi Sancho-Parramon, ${ }^{2}$ and Hrvoje Zorc ${ }^{2}$ \\ ${ }^{1}$ Research Computing Center, Moscow State University, Leninskie Gory, 119991, Moscow, Russia \\ ${ }^{2}$ Rudjer Boskovic Institute, Bijenicka Cesta 54, 10002, Zagreb, Croatia \\ ${ }^{\star}$ Corresponding author: tatiana@srcc.msu.ru
}

Received 28 July 2011; revised 3 October 2011; accepted 3 October 2011; posted 4 October 2011 (Doc. ID 151822); published 15 November 2011

\begin{abstract}
In the present study we determine the optical parameters of thin metal-dielectric films using two different characterization techniques based on nonparametric and multiple oscillator models. We consider four series of thin metal-dielectric films produced under various deposition conditions with different optical properties. We compare characterization results obtained by nonparametric and multiple oscillator techniques and demonstrate that the results are consistent. The consistency of the results proves their reliability. (C) 2011 Optical Society of America

OCIS codes: $\quad 310.3840,310.1620,310.1860,310.3915,310.6860$.
\end{abstract}

\section{Introduction}

In recent years, a number of papers related to characterization and modeling of metal-dielectric thin films have been published [1-16]. The permanently growing interest in the study of thin metal and metal-dielectric films is explained by their specific optical properties, which allow using these films in the design of multilayer structures of special types $[4,6,14,15,17-21]$. In order to design and produce multilayer structures containing metal-dielectric films, it is required to know accurately the optical parameters of such films. In the case of metaldielectric structures, characterization results and design process are interconnected in a more complicated way than in the case of conventional dielectric coatings. The reason is that wavelength dependencies of the optical constants of thin metal-dielectric films are strongly dependent on film thickness $[\underline{5}, \underline{7}, \underline{9}, \underline{12}, \underline{13}, 16,22]$. This means that for design purposes, not one but a series of metal-dielectric films

0003-6935/11/336189-09\$15.00/0

(C) 2011 Optical Society of America with a growing amount of deposited metal is to be carefully characterized.

Recently, an approach to reliable characterization of such metal-dielectric film series was proposed [16]. It was shown in [16] that optical parameters of thin metal films can be reliably determined from ellipsometric measurement data. In [16], a nonparametric model, describing refractive index and extinction coefficient wavelength dependencies $n(\lambda)$ and $k(\lambda)$ as arbitrary smooth functions, was applied.

At the same time, the most commonly used characterization techniques exploit parametric models to describe dispersion behavior of optical constants of metal-dielectric films. The most popular technique is based on a well-known oscillator model [23-25]. However, wavelength dependencies of the optical constants of thin metal-dielectric films in the visible and infrared spectral ranges are quite complicated $[1,7-9,13,26-28]$, and multiple oscillator models have to be used. Because each oscillator is defined by several parameters, a large number of parameters has to be determined in the course of the characterization process using a multiple oscillator model $[23-25,29]$. 
The use of the multiple oscillator model requires knowledge on the material structure, as the optical constants' dispersion is strongly dependent on the composite arrangement: composites consisting of isolated metal clusters are characterized by large absorption within some limited spectral range due to the surface plasmon resonance phenomena $[23,25]$, while composites with a percolated metal phase show metal-like behavior $[25,29]$. Thus, in a general case, the choice of the number of required oscillators and an initial guess for parameter values are not straightforward tasks.

The main advantage of the nonparametric technique in comparison with the multiple oscillator technique is its flexibility and universality: the $n$ and $k$ wavelength dependencies can be determined without special knowledge about thin-film structures.

Nonparametric and multiple oscillator characterization techniques are absolutely different from both the mathematical and physical points of view. Taking into account the complexity of the $n$ and $k$ wavelength dependencies and the possible instability of characterization solutions [16], there are some concerns that these two techniques may provide different characterization results. This would mean that none of the results obtained by the nonparametric technique or by the multiple oscillator technique are considered to be reliable, at least until the correctness of one of them is confirmed by nonoptical characterization methods. It is important, therefore, to compare characterization results obtained by two characterization techniques.

The goal of this paper is to compare two characterization techniques and verify whether they provide close characterization results. Consistency of the obtained results would indicate their reliability. This issue can be very useful for researchers who do not have deep knowledge about metal-dielectric film structures, because then the requirements of the multiple oscillator model are not necessary, as they can obtain the reliable results using the nonparametric model.

In order to provide a sufficient experimental basis for comparing the two characterization techniques, we produced four series of gold and silver metaldielectric samples. In the course of production, we used different deposition parameters for each series. Because of the fact that the structures and optical properties of metal-dielectric films are strongly dependent on deposition parameters, we possess a variety of dispersion dependencies to be determined.

In Section $\underline{2}$ we present our experiment samples and measurement data that we have at our disposal. In Section $\underline{3}$ we briefly describe nonparametric and multiple oscillator characterization techniques. In Section $\underline{4}$ we characterize thin metal-dielectric films using nonparametric and multiple oscillator techniques and compare the results. Our conclusions are given in Section $\underline{5}$.

\section{Experimental Samples and Measurement Data}

In order to provide an experimental basis for comparison of the two characterization techniques, we produced four series of metal-dielectric samples. The samples were prepared by electron beam evaporation in a modified Varian 3117 chamber. The BK7 substrates were positioned onto a rotating fixturing to ensure a uniform thickness of the layers over the sample surface. Base pressure was $4-8 \cdot 10^{-6}$ Torr. The layer mass thickness was controlled by a quartz crystal monitor. Deposition rates were $10 \AA / \mathrm{s}$ for $\mathrm{SiO}_{2}$ and $0.5-1 \AA / \mathrm{s}$ for both $\mathrm{Au}$ and $\mathrm{Ag}$. All the samples had one metal layer embedded between silica layers, so the structure is $\mathrm{SiO}_{2} / \mathrm{metal} / \mathrm{SiO}_{2}$. The mass thickness of the silica layers is $78 \mathrm{~nm}$.

The first and second series consist of the samples where the substrates were preheated to $255^{\circ} \mathrm{C}$ to enhance island growth of the metal [5,22]. The samples within each series differ in the deposited mass thickness of the metal. We refer to these series as S1 with samples $\mathrm{S} 1-1, \ldots, \mathrm{S} 1-5$ containing gold and $\mathrm{S} 2$ with samples $\mathrm{S} 2-1, \ldots, \mathrm{S} 2-5$ containing silver. The corresponding mass thicknesses of metal are presented in the second column of Tables 1 and 2 .

The third series has samples containing silver deposited on substrates that were not preheated. We refer to this series as S3 with samples S3-1,.., S3-5. The mass thicknesses of silver are presented in the second column of Table 3 .

The fourth series includes the samples with silver, all of the same deposited mass thickness, $20 \mathrm{~nm}$. The samples differ by the temperature to what the substrate was preheated, which is presented in Table 4 for each sample. We refer to this series as S4 with samples $\mathrm{S} 4-1, \ldots, \mathrm{S} 4-5$.

Based on the results obtained in our previous publication [16], we provide ellipsometric and photometric data for all samples. The ellipsometric angles $\Psi$ and $\Delta$ and depolarization $q$ for three angles of incidence $45^{\circ}, 55^{\circ}$, and $65^{\circ}$, normal incidence transmittance data $t$ were measured in the spectral range from 285 to $2200 \mathrm{~nm}$ using a Woollam VASE variable angle spectroscopic ellipsometer. Reflectance (R) and transmittance $(\mathrm{T})$ measurements of samples were measured at near-normal incidence in the spectral range from 350 to $1100 \mathrm{~nm}$ using a PerkinElmer Lambda 25 spectrophotometer. Both $T$ and $t$

Table 1. Comparison of Characterization Results for Samples of Series S1

\begin{tabular}{ccccccc}
\hline & \multicolumn{3}{c}{$\begin{array}{c}\text { Nonparametric } \\
\text { Technique }\end{array}$} & & $\begin{array}{c}\text { Multiple Oscillator } \\
\text { Technique }\end{array}$ \\
Sample & $\begin{array}{c}\text { Mass } \\
\text { thickness } \\
(\mathrm{nm})\end{array}$ & $\begin{array}{c}\text { Effective } \\
\text { thickness } \\
(\mathrm{nm})\end{array}$ & \multicolumn{3}{c}{$\begin{array}{c}\text { Effective } \\
\text { thickness } \\
(\mathrm{nm})\end{array}$} & $\mathrm{DF}_{\mathrm{NP}}$ \\
\hline S1-1 & 6.0 & 7.8 & 107.8 & 7.7 & 140.0 \\
S1-2 & 9.0 & 12.4 & 25.0 & 12.4 & 60.0 \\
S1-3 & 12.0 & 15.0 & 30.4 & 14.7 & 77.3 \\
S1-4 & 15.0 & 20.3 & 68.2 & 20.0 & 80.1 \\
S1-5 & 18.0 & 22.7 & 70.8 & 22.6 & 76.5 \\
\hline
\end{tabular}


Table 2. Comparison of Characterization Results for Samples of Series S2

\begin{tabular}{|c|c|c|c|c|c|}
\hline \multirow[b]{2}{*}{ Sample } & \multirow[b]{2}{*}{$\begin{array}{c}\text { Mass } \\
\text { thickness } \\
(\mathrm{nm})\end{array}$} & \multicolumn{2}{|c|}{$\begin{array}{c}\text { Nonparametric } \\
\text { Technique }\end{array}$} & \multicolumn{2}{|c|}{$\begin{array}{c}\text { Multiple Oscillator } \\
\text { Technique }\end{array}$} \\
\hline & & $\begin{array}{c}\text { Effective } \\
\text { thickness } \\
(\mathrm{nm})\end{array}$ & $\mathrm{DF}_{\mathrm{NP}}$ & $\begin{array}{c}\text { Effective } \\
\text { thickness } \\
(\mathrm{nm})\end{array}$ & $\mathrm{DF}_{\mathrm{MO}}$ \\
\hline S2-1 & 6.0 & 12.3 & 44.2 & 12.9 & 49.5 \\
\hline S2-2 & 9.0 & 15.4 & 21.38 & 15.7 & 41.7 \\
\hline S2-3 & 12.0 & 18.5 & 16.6 & 18.7 & 74.5 \\
\hline $\mathrm{S} 2-4$ & 15.0 & 24.3 & 11.2 & 23.5 & 64.4 \\
\hline S2-5 & 18.0 & 25.4 & 15.7 & 27.0 & 94.2 \\
\hline
\end{tabular}

measurements are in good agreement. The transmittance measured with the ellipsometer has a larger experimental error than the transmittance measured with the spectrophotometer. However, we use $t$ in the optical characterization in order to guarantee that all the measurements are taken exactly at the same point and to avoid dealing simultaneously with measurements taken with different instruments.

\section{Description of Characterization Techniques}

\section{A. Nonparametric Characterization Technique}

This technique is based on a nonparametric model, assuming $n(\lambda)$ and $k(\lambda)$ are arbitrary smooth functions $[16,30,31]$. Application of this technique to characterization of thin metal-dielectric films has been demonstrated in $[16,32,33]$. The novelty of the nonparametric characterization technique that we use in the present study is that the depolarization effect caused by back-side reflections was taken into account. Other possible reasons for depolarization (such as layer thickness inhomogeneity, light bandwidth, or beam angular spread) were estimated to be negligible compared to the substrate back-side contribution. Depolarization of the samples is calculated using the relations presented in [34].

In this research, we determine dependencies $n(\lambda)$, $k(\lambda)$ and effective thickness of the metal-dielectric film $\delta$. We also search for thicknesses of silica layers, $d^{(1)}$ and $d^{(2)}$, surrounding metal-dielectric films. The discrepancy function estimating deviations of model spectral characteristics from measured characteristics is written in the following way:
Table 3. Comparison of Characterization Results for Samples of Series S3

\begin{tabular}{|c|c|c|c|c|c|}
\hline \multirow[b]{2}{*}{ Sample } & \multirow[b]{2}{*}{$\begin{array}{l}\text { Mass } \\
\text { thickness } \\
\quad(\mathrm{nm})\end{array}$} & \multicolumn{2}{|c|}{$\begin{array}{l}\text { Nonparametric } \\
\text { Technique }\end{array}$} & \multicolumn{2}{|c|}{$\begin{array}{l}\text { Multiple Oscillator } \\
\text { Technique }\end{array}$} \\
\hline & & $\begin{array}{c}\text { Effective } \\
\text { thickness } \\
(\mathrm{nm})\end{array}$ & $\mathrm{DF}_{\mathrm{NP}}$ & $\begin{array}{c}\text { Effective } \\
\text { thickness } \\
(\mathrm{nm})\end{array}$ & $\mathrm{DF}_{\mathrm{MO}}$ \\
\hline S3-1 & 6.0 & 8.4 & 31.9 & 8.8 & 35.2 \\
\hline S3-2 & 9.0 & 8.6 & 37.2 & 9.3 & 45.8 \\
\hline S3-3 & 12.0 & 10.8 & 20.0 & 10.4 & 58.7 \\
\hline S3-4 & 15.0 & 12.0 & 19.4 & 12.2 & 53.8 \\
\hline S3-5 & 18.0 & 13.7 & 8.1 & 13.4 & 14.8 \\
\hline
\end{tabular}

Table 4. Comparison of Characterization Results for Samples of Series S4

\begin{tabular}{|c|c|c|c|c|c|c|}
\hline \multirow[b]{2}{*}{ Sample } & \multirow[b]{2}{*}{$\begin{array}{c}\text { Substrate } \\
\text { temp. } \\
\left({ }^{\circ} \mathrm{C}\right)\end{array}$} & \multirow[b]{2}{*}{$\begin{array}{c}\text { Mass } \\
\text { thickness } \\
\quad(\mathrm{nm})\end{array}$} & \multicolumn{2}{|c|}{$\begin{array}{c}\text { Nonparametric } \\
\text { Technique }\end{array}$} & \multicolumn{2}{|c|}{$\begin{array}{l}\text { Multiple } \\
\text { Oscillator } \\
\text { Technique }\end{array}$} \\
\hline & & & $\begin{array}{c}\text { Effective } \\
\text { thickness } \\
\quad(\mathrm{nm})\end{array}$ & $\mathrm{DF}_{\mathrm{NP}}$ & $\begin{array}{c}\text { Effective } \\
\text { thickness } \\
\quad(\mathrm{nm})\end{array}$ & $\mathrm{DF}_{\mathrm{MO}}$ \\
\hline S4-1 & 45 & 20.0 & 15.0 & 15.3 & 13.8 & 131.6 \\
\hline S4-2 & 70 & 20.0 & 16.6 & 14.2 & 16.2 & 100.6 \\
\hline S4-3 & 100 & 20.0 & 20.0 & 12.8 & 20.6 & 88.2 \\
\hline S4-4 & 115 & 20.0 & 21.9 & 16.4 & 23.4 & 94.9 \\
\hline S4-5 & 225 & 20.0 & 25.4 & 13.1 & 25.7 & 87.7 \\
\hline
\end{tabular}

where $\theta_{1}=45^{\circ}, \theta_{2}=55^{\circ}, \theta_{3}=65^{\circ} ;\left\{\lambda_{j}\right\}$ is the wavelength grid in the spectral range from 285 to $2200 \mathrm{~nm} ; \Psi(\cdot)$ and $\Delta(\cdot)$ are the model ellipsometric angles of the sample; $q(\cdot)$ is the model depolarization; $t(\cdot)$ is the model transmittance of the sample; $\hat{\Psi}, \hat{\Delta}, \hat{q}$, $\hat{t}$ are corresponding measured spectral characteristics; and $\Delta \Psi_{j}, \Delta \Delta_{j}, \Delta q_{j}$, and $\Delta t_{j}$ are experimental errors recorded by Woollam VASE ellipsometer. In Eq. (1) $n^{\prime \prime}(\lambda)$ and $k^{\prime \prime}(\lambda)$ denote the numerical secondorder derivatives of the refractive index and extinction coefficient and $\alpha_{1}$ and $\alpha_{2}$ are weight factors controlling the correlation between the smoothness demand and demand on a good fit of measurement data by model data. $M=10$ is the total number of measurement data arrays: two ellipsometric angles measured at three different angles of incidence;

$$
\begin{aligned}
D F^{2}= & \frac{1}{190 M}\left\{\sum_{k=1}^{3} \sum_{j=1}^{190}\left[\frac{\Psi\left(n\left(\lambda_{j}\right), k\left(\lambda_{j}\right), \delta, \lambda_{j}, d^{(1)}, d^{(2)}, \theta_{k}\right)-\hat{\Psi}\left(\lambda_{j}, \theta_{k}\right)}{\Delta \Psi_{j}}\right]^{2}\right. \\
& +\sum_{k=1}^{3} \sum_{j=1}^{190}\left[\frac{\Delta\left(n\left(\lambda_{j}\right), k\left(\lambda_{j}\right), \delta, \lambda_{j}, d^{(1)}, d^{(2)}, \theta_{k}\right)-\hat{\Delta}\left(\lambda_{j}, \theta_{k}\right)}{\Delta \Delta_{j}}\right]^{2}+\sum_{k=1}^{3} \sum_{j=1}^{190}\left[\frac{q\left(n\left(\lambda_{j}\right), k\left(\lambda_{j}\right), \delta, \lambda_{j}, d^{(1)}, d^{(2)}, \theta_{k}\right)-\hat{q}\left(\lambda_{j}, \theta_{k}\right)}{\Delta q_{j}}\right]^{2} \\
& \left.+\sum_{j=1}^{190}\left[\frac{t\left(n\left(\lambda_{j}\right), k\left(\lambda_{j}\right), \delta, \lambda_{j}, d^{(1)}, d^{(2)}\right)-\hat{t}\left(\lambda_{j}\right)}{\Delta t_{j}}\right]^{2}+\alpha_{1} \sum_{j=1}^{190}\left[n^{\prime \prime}\left(\lambda_{j}\right)\right]^{2}+\alpha_{2} \sum_{j=1}^{190}\left[k^{\prime \prime}\left(\lambda_{j}\right)\right]^{2}\right\}
\end{aligned}
$$


depolarization is also measured at three incidence angles and normal incidence transmittance.

For all samples, in the course of the characterization procedure, the parameters $\alpha_{1}$ and $\alpha_{2}$ were equal to each other and were gradually decreased from $1 \cdot 10^{-2}$ to $1 \cdot 10^{-8}$ to reach a good fitting of the measurement data on the one hand, and to keep the stability of the solutions on the other hand.

The characterization algorithm based on minimization of the discrepancy function (1) is incorporated in OptiRE module of OptiLayer thin-film software [35]. We used this algorithm in our characterization process. where $\varepsilon(E)=(n(E)-i k(E))^{2}$ is the dimensionless dielectric function, $E=1240 / \lambda, \varepsilon_{\infty}$ is the value of the real part of the dielectric function at very large photon energies, and $N$ is the number of oscillators. Each oscillator is described by three parameters: $A_{i}$ is the dimensionless amplitude of the $i$ th oscillator, $B_{i}$ and $E_{c, i}$ are the broadening and center energy of the $i$ th oscillator, respectively; they have units of electron volts. $P$ is the Cauchy principal value of the integral.

In order to estimate deviations between the model and measurement spectral characteristics, we calculate discrepancy function in the following way [38]:

$$
\begin{aligned}
\mathrm{DF}^{2}= & \frac{1}{190 \cdot M-p}\left(\sum_{k=1}^{3} \sum_{j=1}^{190}\left[\frac{\Psi\left(A, B, E_{c}, E_{j}, \delta, d^{(1)}, d^{(2)}, \theta_{k}\right)-\hat{\Psi}\left(E_{j}, \theta_{k}\right)}{\Delta \Psi_{j}}\right]^{2}\right. \\
& +\sum_{k=1}^{3} \sum_{j=1}^{190}\left[\frac{\Delta\left(A, B, E_{c}, E_{j}, \delta, d^{(1)}, d^{(2)}, \theta_{k}\right)-\hat{\Delta}\left(E_{j}, \theta_{k}\right)}{\Delta \Delta_{j}}\right]^{2}+\sum_{k=1}^{3} \sum_{j=1}^{190}\left[\frac{q\left(A, B, E_{c}, E_{j}, \delta, d^{(1)}, d^{(2)}, \theta_{k}\right)-\hat{q}\left(E_{j}, \theta_{k}\right)}{\Delta q_{j}}\right]^{2} \\
& \left.+\sum_{j=1}^{190}\left[\frac{t\left(A, B, E_{c}, E_{j}, \delta, d^{(1)}, d^{(2)}\right)-\hat{t}\left(E_{j}\right)}{\Delta t_{j}}\right]^{2}\right)
\end{aligned}
$$

\section{B. Multiple Oscillator Characterization Technique}

The multiple oscillator technique is based on the multiple oscillator model. In the frame of this model we describe the $n(\lambda)$ and $k(\lambda)$ dispersion behavior using Gauss oscillators. Although the classical Lorentz oscillators are widely used [12], it has been shown that Gauss oscillators are more flexible and provide better data fits than Lorentz oscillators $[25,36]$. The reason is that in random metaldielectric composites, particles present a size and shape distribution and one may expect that the absorption is due to a distribution of resonances rather than to a single resonance. Assuming a Gaussian distribution of resonances and that the width of this distribution is large enough, the imaginary part of the dielectric function may present a Gaussian line shape $[23,37]$. In this case the dielectric function is written in the following way:

$$
\begin{aligned}
& \varepsilon(E)=\varepsilon_{\infty}+\sum_{i=1}^{N} \varepsilon_{\mathrm{Gauss}, i}(E), \\
& \operatorname{Im} \varepsilon_{\mathrm{Gauss}, i}(E)=A_{i} \exp \left[-\left(\frac{E-E_{c, i}}{B_{i}}\right)^{2}\right] \\
& -A_{i} \exp \left[-\left(\frac{E+E_{c, i}}{B_{i}}\right)^{2}\right], \\
& \operatorname{Re} \varepsilon_{\mathrm{Gauss}, i}(E)=\frac{2}{\pi} P \int_{0}^{+\infty} \frac{\xi \operatorname{Im} \varepsilon_{\mathrm{Gauss}, i}(E)}{\xi^{2}-E^{2}} d \xi,
\end{aligned}
$$

where $E_{j}=1240 / \lambda_{j}$. Here $A=\left\{A_{1}, \ldots, A_{N}\right\}, \quad B=$ $\left\{B_{1}, \ldots, B_{N}\right\}, E_{c}=\left\{E_{c, 1}, \ldots, E_{c . N}\right\}$ are sets of unknown model parameters and $p$ is the total number of optimized parameters.

The characterization algorithm based on minimization of DF in Eq. (3) is incorporated in Woollam WVASE32 software [38]. As mentioned before, the dispersion of optical constants of metal-dielectric composites is strongly dependent on the composite microstructure and an initial guess of the number and parameters of required oscillators is not straightforward. Thus, our characterization procedure has been as follows: first we start with a wavelength-by-wavelength inversion of the optical constants of the metal-dielectric composite layer, fixing the thicknesses of the layers to the design values. From the obtained optical constants, the number of oscillators parameters can be estimated by analyzing the presence of peaks in the imaginary part of the dielectric function. Initial values of the parameters can be obtained by fitting the optical constants determined wavelength-by-wavelength to a fixed number of oscillators. This analysis is performed using the "GenOsc" function of the WVASE32 software. Once an initial guess of the oscillator parameters is obtained, the final fitting is performed by simultaneously optimizing the oscillator parameters and the thicknesses of layers.

In our characterization procedure we use six oscillators to approximate $n$ and $k$ wavelength dependencies, so $N=6$. Therefore, the number of unknown parameters $p$ in Eq. (3) is 22: three parameters for 
each oscillator, one parameter $\varepsilon_{\infty}$, and thicknesses $\delta$, $d^{(1)}, d^{(2)}$.

\section{Characterization of Experimental Samples}

It has been mentioned in the Introduction that in the case of metal-dielectric film characterization, not one but a series of films with increasing thicknesses is to be performed. In order to obtain reliable results, two important issues should be taken into account in the course of the characterization process. The first issue is that the samples are to be ranged with respect to effective thickness $\delta$. This ranging cannot be done only based on mass thickness values, because these values are delivered by a quartz crystal monitor and some errors are possible. In order to range the samples in a proper sequence according to $\delta$ values, $a$ priori information should be used. In our research we use spectrophotometric data $R$ and $T$ to range samples in each series from minimal to maximal $\delta$ values. For each series we give a physical explanation of how to use spectrophotometric data for this purpose.

The second issue related to the characterization of the sample series is that the obtained patterns of wavelength dependencies of optical constants are expected to form a regular physically sensible sequence. For example, physical explanation of resonance positions and amplitudes of $\mathrm{Ag}-\mathrm{SiO}_{2}$ films was provided in [16].

\section{A. Characterization of Series S1}

According to our previous investigations [36] and taking into account the deposition conditions, we expect that the films of the first series consist of nanometric and well-separated $\mathrm{Au}$ islands, with the island size increasing and the island density decreasing with the increase of the deposited metal amount.

In order to check that higher values of effective thickness correspond to higher values of mass thickness, we compare total losses $L(\lambda)=100 \%-R(\lambda)-$ $T(\lambda)$ in samples $\mathrm{S} 1-1, \ldots, \mathrm{S} 1-5$ of this series (see Fig. 1). The spectral behavior of $L(\lambda)$ for all the sam-

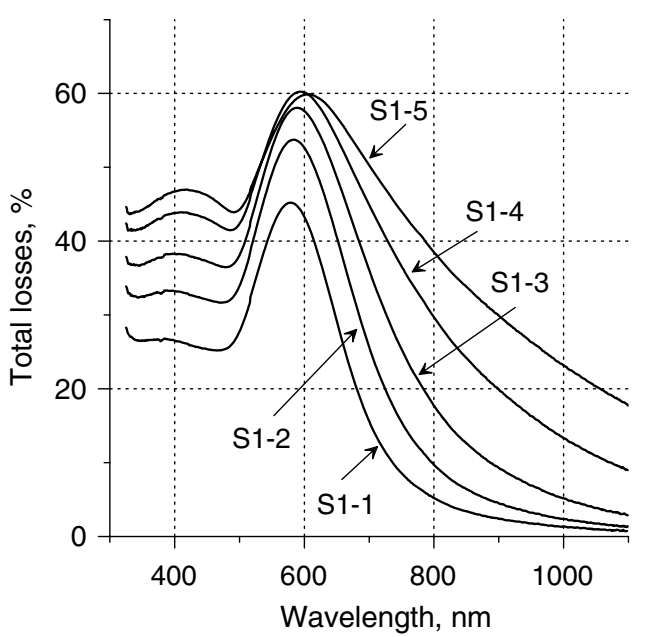

Fig. 1. Comparison of total losses in samples of series S1. The labels $\mathrm{S} 1-1, \ldots, \mathrm{S} 1-5$ indicate the corresponding samples. ples is characterized by a broad peak centered at $550-600 \mathrm{~nm}$, corresponding to the surface plasmon resonance. The intensity of this resonance grows as the amount of deposited metal increases, while its position slightly shifts to longer wavelengths. This behavior can be understood as an increase of the concentration of metal in the metal-dielectric composite layer [39]. In addition, there is a secondary peak, centered at approximately $400 \mathrm{~nm}$, corresponding to the interband absorption in gold $[26,28]$. The intensity of this peak should become larger as the total amount of metal in the composite layer increases.

We perform characterization of the samples S1-1,.., S1-5 using the nonparametric and multiple oscillator techniques described in the previous section. In order to compare the achieved closeness between the measured and model spectral characteristics, we compare the values, $\mathrm{DF}_{\mathrm{NP}}$ and $\mathrm{DF}_{\mathrm{MO}}$, obtained by nonparametric and multiple oscillator techniques, respectively. Both values are calculated as weighted rms deviations of model data from experimental data. These values allow one to compare fittings achieved in the course of the characterization procedure. We compare $\mathrm{DF}_{\mathrm{NP}}$ and $\mathrm{DF}_{\mathrm{MO}}$ values in the fourth and sixth columns of Table 1 and observe that $\mathrm{DF}_{\mathrm{MO}}$ are larger than $\mathrm{DF}_{\mathrm{NP}}$ for all the samples of series $\mathrm{S} 1$. This indicates that the nonparametric model might yield better fitting than the multiple oscillator model.

In Fig. 2 we compare the $n$ and $k$ wavelength dependencies determined by nonparametric (solid curves) and multiple oscillator (dashed curves) models. Effective thicknesses of $\mathrm{Au}-\mathrm{SiO}_{2}$ films obtained by two techniques are presented in Table 1 . It should be noted here that in the course of characterization process we utilize measurement data from the spectral range of 285 to $2200 \mathrm{~nm}$, but in Fig. 2 we compare $n, k$ in the spectral range from 285 to $1100 \mathrm{~nm}$. This is due to the fact that the main features of $n$ and $k$ curves are observed in the visible and near-infrared spectral ranges. In Fig. $\underline{2}$ and Table $\underline{1}$, one can observe a remarkable agreement between characterization results obtained by two absolutely different techniques. In the range of 1100 to $2175 \mathrm{~nm}$, obtained refractive indices and extinction coefficients for all samples are very close for both characterization techniques.

From Fig. 2 it is obvious that the $n$ and $k$ curves form a physically sensible regular sequence with respect to the effective thickness of $\mathrm{Au}-\mathrm{SiO}_{2}$ films. The dispersion of the effective optical constants is mainly determined by the surface plasmon resonance, which increases in intensity and redshifts as the amount of metal increases, as already discussed above. The refractive index shows the typical dispersion around a resonance. In addition to the surface plasmon resonance, a secondary and less intense resonance is evidenced at shorter wavelengths, connected to the interband transitions in gold. 

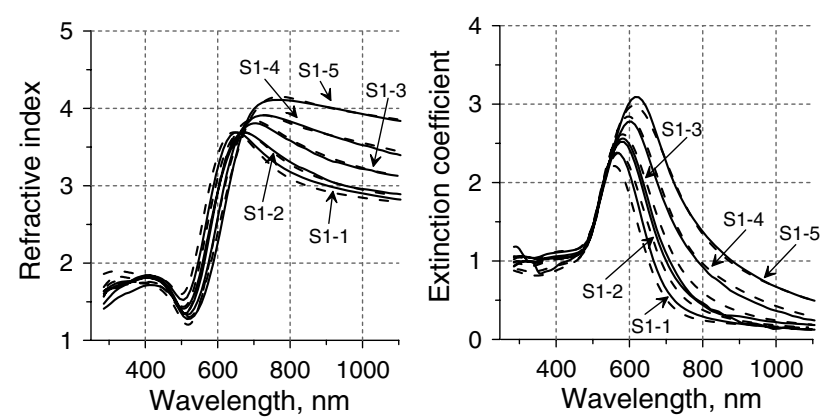

Fig. 2. Wavelength dependencies of refractive indices and extinction coefficients of $\mathrm{Au}-\mathrm{SiO}_{2}$ composite films deposited on preheated substrates (series S1). The labels S1-1,.., S1-5 indicate the corresponding samples.

\section{B. Characterization of Series S2}

The films of the second series are characterized by well-separated Ag islands, and their optical properties are identified by the surface plasmon resonance that increases in intensity and redshifts as the amount of deposited Ag increases [39].

As in the case with gold-silica films, in order to check that higher values of effective thickness of $\mathrm{Ag}-\mathrm{SiO}_{2}$ films correspond to higher values of mass thicknesses, we compare total losses in samples $\mathrm{S} 2-1, \ldots, \mathrm{S} 2-5$ of this series (see Fig. 3). The absorption behavior of this series closely resembles the one of the S1 series and obeys the same physical phenomena: an increase and redshift of the surfaceplasmon-resonance-related absorption that can be associated to the larger amount of metal in the metal-dielectric composite.

In Fig. 4 we compare $n(\lambda)$ and $k(\lambda)$ obtained using nonparametric (solid curves) and multiple oscillator (dashed curves) techniques. Effective thicknesses of $\mathrm{Ag}-\mathrm{SiO}_{2}$ films obtained by two techniques are presented in Table $\underline{2}$. In Fig. $\underline{4}$ and Table $\underline{2}$, a good agreement between characterization results obtained by two different techniques is observed. As for series $\mathrm{S} 1$, a comparison of $\mathrm{DF}_{\mathrm{NP}}$ and $\mathrm{DF}_{\mathrm{MO}}$ values

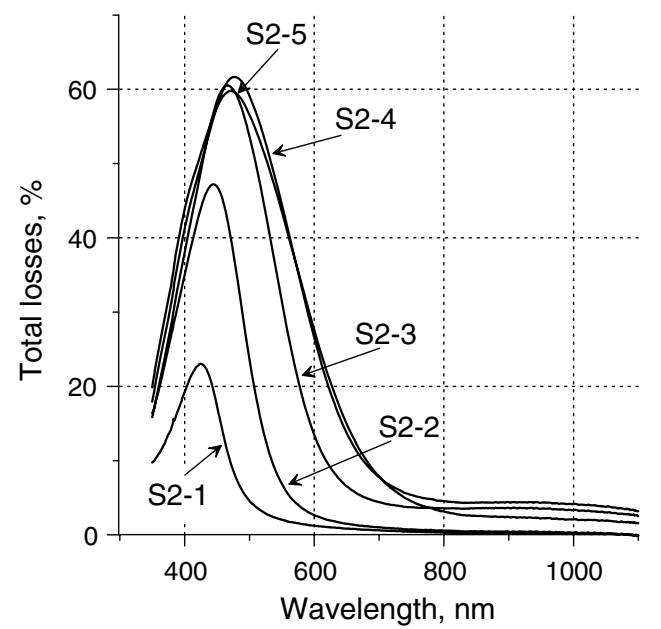

Fig. 3. Comparison of total losses in samples of series S2. The labels $\mathrm{S} 2-1, \ldots, \mathrm{S} 2-5$ indicate the corresponding samples.
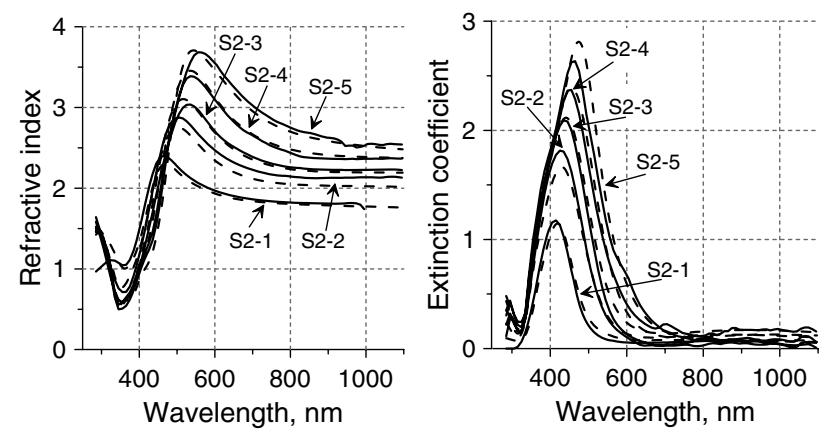

Fig. 4. Wavelength dependencies of refractive indices and extinction coefficients of $\mathrm{Ag}-\mathrm{SiO}_{2}$ composite films deposited on preheated substrates (series S2). The labels S2-1,.., S2-5 indicate the corresponding samples.

presented in Table $\underline{2}$ indicates better fittings achieved by the nonparametric model.

It is seen from Fig. $\underline{4}$ that the $n$ and $k$ curves form a physically sensible sequence with the growing effective thickness of $\mathrm{Ag}-\mathrm{SiO}_{2}$ films. The same as for series $\mathrm{S} 1$, the wavelength dependence of the effective optical constants is dominated by the surface plasmon resonance.

\section{Characterization of Series S3}

In this case, the interpretation of the absorption spectra is more complex, and two different phenomena are involved: the surface plasmon resonance of isolated metal particles and the Drude-like absorption of the percolated metal network. In this case, we range samples based on analysis of the measured reflectance data (see Fig. 5). The increase of reflectance at longer wavelengths is related to the increase of the Drude-like absorption; i.e., the metal phase becomes more percolated. On the other hand, the increase of reflectance below $400 \mathrm{~nm}$ is related to the increase of absorption due to interband transitions, indicating a larger amount of metal in the composite. These observations suggest that by decreasing the

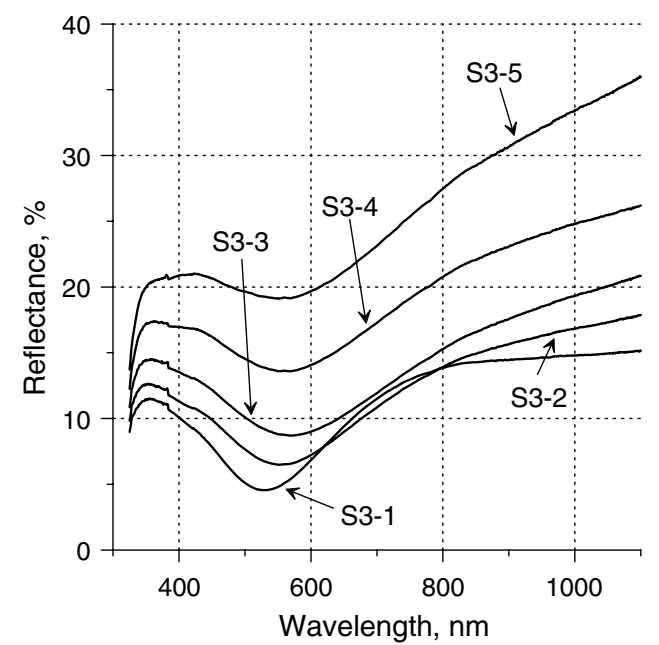

Fig. 5. Comparison of measured reflectance data of samples of series S3. The labels S3-1, ., S3-5 indicate the corresponding samples. 
deposition temperature, the metal is deposited more compactly and the composite has a larger metal fraction.

In Fig. $\underline{6}$ we compare wavelength dependencies of refractive indices and extinction coefficients determined by nonparametric (solid curves) and multiple oscillator (dashed curves) techniques. Effective thicknesses of the films found by two techniques are compared in Table 3. It is seen from Fig. 6 and Table 3 that the obtained characterization results are consistent. As in the previous cases $\mathrm{DF}_{\mathrm{NP}}<\mathrm{DF}_{\mathrm{MO}}$ for all samples of this series.

It is seen from Fig. 6 that the refractive indices and extinction coefficients of films in samples $\mathrm{S} 3-1, \ldots, \mathrm{S} 3-5$ increase with growing effective thickness of $\mathrm{Ag}-\mathrm{SiO}_{2}$ films. At small values of the mass thickness, the layer is basically formed by isolated metal particles and the effective optical behavior is dominated by the surface plasmon resonance peak. In this case, the resonance is broader and less intense than in the $\mathrm{S} 2$ series because the islands grow more elliptical and denser at unheated substrates for the same amount of deposited material [39]. As the amount of metal increases, percolation among the islands starts and the metal-like behavior, characterized by strong infrared absorption, becomes evident in the effective optical constants.

According to our previous studies of thin metal compact layers, deposited by the same technique and conditions [25], the real film thickness is approximately $2 / 3$ of the mass thickness values (i.e., the values provided by the quartz crystal monitor). When this correction is applied to the mass thicknesses given in Table 3 , then for the composites with the largest mass thicknesses, the ratio to the effective thickness is close to 1 , indicating that the metal is forming an almost compact layer.

\section{Characterization of Series S4}

Samples of this series can be ranged on the basis of analysis of total losses, which are shown in Fig. 7. At hotter substrates, the island formation is enhanced, resulting in larger, more spherical, and well-isolated particles, characterized by a narrow surface plasmon resonance. On the other hand, as the substrate tem-
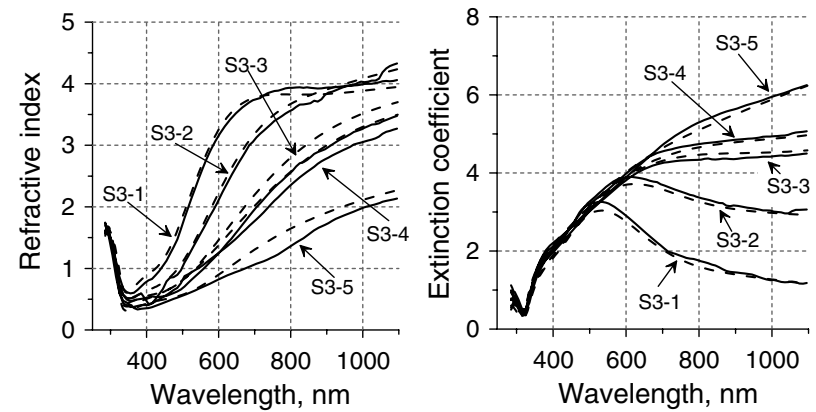

Fig. 6. Wavelength dependencies of refractive indices and extinction coefficients of $\mathrm{Ag}-\mathrm{SiO}_{2}$ composite films deposited on unheated substrates (series S3). The labels S3-1, .., S3-5 indicate the corresponding samples.

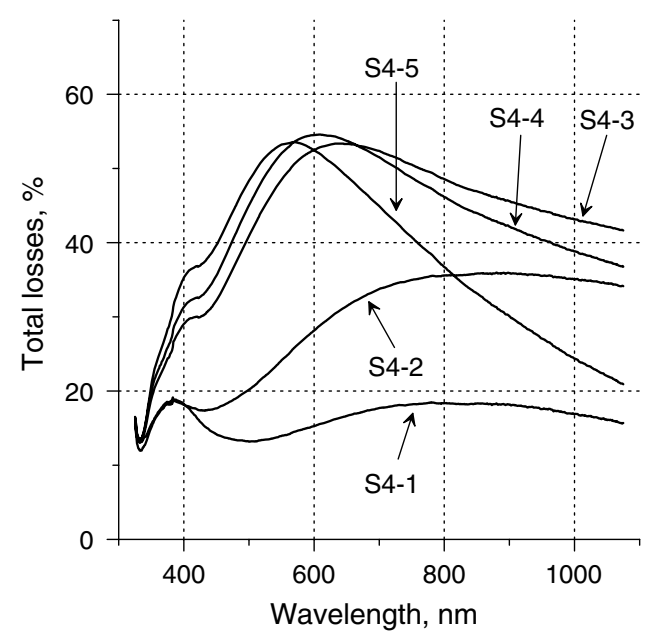

Fig. 7. Comparison of total losses in samples of series S4. The labels $\mathrm{S} 4-1, \ldots, \mathrm{S} 4-5$ indicate the corresponding samples.

perature decreases the islands become smaller, more elliptical, and with some degree of interconnection. In this case, the surface plasmon resonance becomes broader and redshifted [25]. Therefore, one may expect that the samples deposited at hotter substrates will have a larger effective thickness than those deposited on unheated substrates.

In Fig. 8 we compare $n(\lambda)$ and $k(\lambda)$ values determined by nonparametric (solid curves) and multiple oscillator (dashed curves) techniques. Effective thicknesses of the films found by two techniques are compared in Table 4 . It is seen from Fig. 8 and Table 4 that, in general, characterization results obtained by two different techniques are in agreement, but differences in $n, k$, and $\delta$ values are observed. In Table 4 we see that the $\mathrm{DF}_{\mathrm{MO}}$ values are significantly higher than the $\mathrm{DF}_{\mathrm{NP}}$ values. These large differences indicate that fittings obtained using the nonparametric model are essentially better than fittings achieved by the multiple oscillator model. There are several possible explanations of this fact. First, in the course of the characterization procedure in the case of the multiple oscillator technique, the optical constants $n$ and $k$ are interconnected [see Eq. (2)], and in the case of the nonparametric model they vary independently. Second, the dependencies of the optical parameters of the films in series $\mathrm{S} 4$ are to be described by
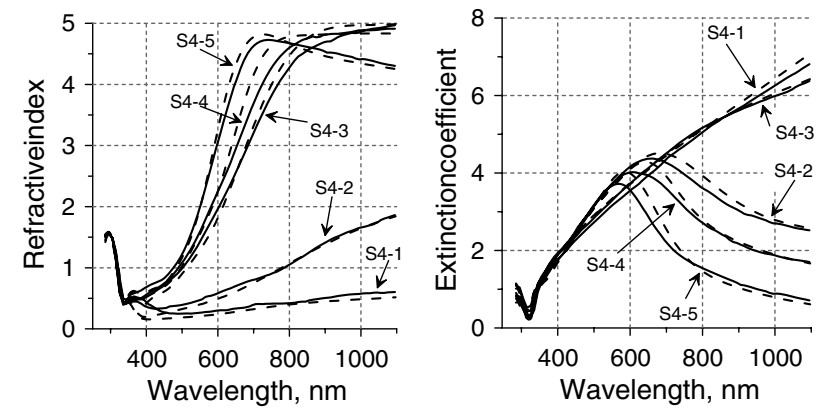

Fig. 8. Wavelength dependencies of refractive indices and extinction coefficients of $\mathrm{Ag}-\mathrm{SiO}_{2}$ composite films (series $\mathrm{S} 4$ ). The labels $\mathrm{S} 4-1, \ldots, \mathrm{S} 4-5$ indicate the corresponding samples. 
more complicated dispersion models having not six but more oscillators. This means that the number of unknown parameters will be increased and, therefore, instability of their determination will grow. At the same time, introduction of more oscillators may not have a physical sense. Third, due to the probable anisotropic structure of metal island films, ellipsometric data obtained at incidence angles between $45^{\circ}$ and $65^{\circ}$ do not need to be consistent with normal incidence transmittance data. This may lead to an additional increase of the discrepancy in the case of the multiple oscillator technique. These three explanations are valid also for series S1-S3, although we observe much smaller differences in the characterization results there.

It is seen from Fig. 8 that the refractive indices and extinction coefficients of films in samples $\mathrm{S} 4-1, \ldots$, S4-5 increase with the increasing temperature of the substrate. In addition to this, we observe in Table 4 that higher ratios of effective and mass thicknesses correspond to higher temperatures. The obtained dispersion of the optical constants reflects the previous comments: the samples deposited on preheated substrates are characterized by a narrow surface plasmon resonance while the composites at unheated substrates show a much broader resonance. In addition, the effective absorption of composites deposited at a low temperature is larger than that of composites deposited at preheated substrates, as the fraction of the metal in the composite is larger. The increase of metal concentration in the composite with lowering the deposition temperature is also confirmed by the decrease of the effective thickness.

\section{Conclusions}

In this work we applied nonparametric and multiple oscillator characterization techniques to the determination of effective optical parameters of metaldielectric films. In order to provide a broad variety of $n$ and $k$ dispersion dependencies to be determined, we produced four series of samples of metaldielectric films using different deposition conditions. We performed characterization of the samples and compared results. This comparison showed that optical parameters determined by two techniques are consistent for all samples. The differences between the optical parameters determined using the two techniques are correlated with differences in fittings achieved in the course of the corresponding characterization procedures. For all samples, fittings provided by nonparametric technique were better than fittings achieved by multiple oscillator technique. This is explained by the higher flexibility of the nonparametric technique in comparison with the multiple oscillator technique.

Our study benefits from the presence of a diverse experimental basis, including a variety of samples and combinations of measurement data. We considered films with different optical properties and demonstrated consistency of characterization results for all films, we state that the nonparametric and multiple oscillator techniques provide reliable characterization results for any types of metal-dielectric films. This work is of a practical importance for researchers who do not have a deep knowledge of metal-dielectric films structures that is necessary for obtaining physically sensible results by the multiple oscillator technique. These researchers can use a more flexible and universal nonparametric technique and be guaranteed reliability of the characterization results.

This work is supported by the National Foundation of Science, Higher Education and Technological development of the Republic of Croatia and the Russian Foundation for Basic Research (RFBR), project 1007-00480-a.

\section{References}

1. A. Leitner, Z. Zhao, H. Brunner, F. Aussenegg, and A. Wokaun, "Optical properties of a metal island film close to a smooth metal surface," Appl. Opt. 32, 102-110 (1993).

2. O. Stenzel, A. Stendal, M. R. Röder, S. Wilbrandt, D. Drews, T. Werninghaus, C. von Borczyskowski, and D. R. T. Zahn, "Localized plasmon excitation in metal nanoclusters as a tool to study thickness-dependent optical properties of copper phthalocyanine ultrathin films," Nanotechnology 9, 6-19 (1998).

3. O. Stenzel, A. Stendal, M. Röder, and C. von Borczyskowski, "Tuning of the plasmon absorption frequency of silver and indium nanoclusters via thin amorphous silicon films," Pure Appl. Opt. 6, 577-588 (1997).

4. A. Stepanov, "Optical transmission of dielectric layers with metallic nanoparticles inhomogeneously distributed over the sample thickness," Opt. Spectrosc. 91, 815-819 (2001).

5. N. Kaiser, "Review of the fundamentals of thin-film growth," Appl. Opt. 41, 3053-3060 (2002).

6. P. Heger, O. Stenzel, and N. Kaiser, "Design and fabrication of selective thin film absorbers on the basis of silver island films," Vacuum's Best VIP 18, 53-56 (2006).

7. A. de Vries, E. Kooij, H. Wormeester, A. Mewe, and B. Poelsema, "Ellipsometric study of percolation in electroless deposited silver films,” J. Appl. Phys. 101, 053703 (2007).

8. A. Lehmuskero, M. Kuittinen, and P. Vahimaa, "Refractive index and extinction coefficient dependence of thin $\mathrm{Al}$ and $\mathrm{Ir}$ films on deposition technique and thickness," Opt. Express 15, 10744-10752 (2007).

9. T. Iwata and G. Komoda, "Measurement of complex refractive indices of metals at several wavelengths by frustrated total internal reflection due to surface plasmon resonance," Appl. Opt. 47, 2386-2391 (2008).

10. I. Hooper and J. Sambles, "Some considerations on the transmissivity of thin metal films," Opt. Express 16, 17249-17257 (2008).

11. Y. Jourlin, S. Tonchev, T. Tishchenko, C. Pedri, C. Veillas, O. Parriaux, A. Last, and Y. Lacroute, "Spatially and polarization resolved plasmon mediated transmission through continuous metal films," Opt. Express 17, 12155-12160 (2009).

12. M. Hövel, B. Gompf, and M. Dressel, "Dielectric properties of ultrathin metal films around the percolation threshold," Phys. Rev. B 81, 035402 (2010).

13. A. Nabok and S. A. Tsargorodskaya, "Ellipsometry study of ultra thin layers of evaporated gold," Phys. Status Solidi C 5, 1150-1155 (2008).

14. W. Chen, M. Thoreson, S. Ishii, A. Kildishev, and V. Shalaev, "Ultra-thin ultra-smooth and low-loss silver films on a germanium wetting layer," Opt. Express 18, 5124-5134 (2010). 
15. H. Zorc, M. Lončarić, J. Sancho-Parramon, and V. Janicki, "Use of gold island films in design of reflectors with luminosity," in Optical Interference Coatings on CD-ROM (Optical Society of America, 2010), paper TuD8.

16. T.Amotchkina, V.Janicki,J.Sancho-Parramon,A.Tikhonravov, M. Trubetskov, and H. Zorc, "General approach to reliable characterization of thin metal films," Appl. Opt. 50, 1453-1464 (2011).

17. J. Dobrowolski, S. Browning, M. Jacobson, and M. Nadal, "2007 OSA Topical Meeting on Optical Interference Coatings: manufacturing problem," Appl. Opt. 47, C231-C245 (2008).

18. J. Dobrowolski, S. Browning, M. Jacobson, and M. Nadal, "2004 Optical Society of America's Topical Meeting on Optical Interference Coatings: manufacturing problem," Appl. Opt. 45, 1303-1311 (2006).

19. J. Dobrowolski, L. Li, and R. Kemp, "Metal/dielectric transmission interference filters with low reflectance. 1. design," Appl. Opt. 34, 5673-5683 (1995).

20. N. Kaiser, "Some fundamentals of optical thin film growth," in Optical Interference Coatings, N. Kaiser and H. K. Pulker, eds. (Springer-Verlag, 2003), pp. 59-80.

21. P. Ma, F. Lin, and J. Dobrowolski, "Design and manufacture of metal-dielectric long wavelength cut-off filters," in Optical Interference Coatings on CD-ROM, OSA Technical Digest Series (Optical Society of America, 2010), paper MA9.

22. U. Kreibig and M. Vollmer, Optical Properties of Metal Clusters (Springer, 1995).

23. O. Stenzel, S. Wilbrandt,A. Stendal, U. Beckers, K. Voigtsberger, and C. von Borczyskowski, "The incorporation of metal clusters into thin organic dye layers as a method for producing strongly absorbing composite layers: an oscillator model approach to resonant metal cluster absorption,” J. Phys. D 28, 2154-2162 (1995).

24. A. B. Djurisic, T. Fritz, and K. Leo, "Modelling the optical constants of organic thin films: impact of the choice of objective function," J. Opt. A 2, 458-464 (2000).

25. J. Sancho-Parramon, V. Janicki, and H. Zorc, "Tuning the effective dielectric function of thin film metal-dielectric composites by controlling the deposition temperature," J. Nanophoton. 5, 051805 (2011).
26. E. D. Palik, Handbook of Optical Constants of Solids (Academic, 1985).

27. O. Stenzel, The Physics of Thin Film Optical Spectra (Springer-Verlag, 2005).

28. P. Johnson and R. Christy, "Optical constants of the noble metals," Phys. Rev. B 6, 4370-4379 (1972).

29. J. Bulir, M. Novotny, A. Lynnykova, and J. Lancok, "Preparation of nanostructured ultrathin silver layer," J. Nanophoton. 5, 051511 (2011).

30. O. Stenzel and R. Petrich, "Flexible construction of error functions and their minimization: application to the calculation of optical constants of absorbing or scattering thin-film materials from spectrophotometric data," J. Phys. D 28, 978-989 (1995).

31. A. V. Tikhonravov, M. K. Trubetskov, J. Hrdina, and J. Sobota, "Characterization of quasi-rugate filters using ellipsometric measurements," Thin Solid Films 277, 83-89 (1996).

32. A. V. Tikhonravov, M. K. Trubetskov, O. F. Prosovskiy, and M. A. Kokarev, "Optical characterization of thin metal films," in Optical Interference Coatings (Optical Society of America, 2007), paper WDPDP2.

33. A. Tikhonravov, M. Trubetskov, T. Amotchkina, M. Kokarev, I. Kozlov, V. Zhupanov, E. Kluev, and O. Prosovskiy, "Optical coatings containing well-controlled few nanometer thick metal layers," in Nanofair 2008. New Ideas for Industry (WDI Wissensforum GmbH, 2008), pp. 171-174.

34. H. Fujuwara, Spectroscopic Ellipsometry: Principles and Applications (Wiley, 2007).

35. A. V. Tikhonravov and M. K. Trubetskov, OptiLayer thin film software, http://www.optilayer.com.

36. M. Lončarić, J. Sancho-Parramon, and H. Zorc, "Optical properties of gold islands films-a spectroscopic ellipsometry study," Thin Solid Films 519, 2946-2950 (2011).

37. A. Franke, A. Stendal, O. Stenzel, and C. von Borczyskowski, "Gaussian quadrature approach to the calculation of the optical constants in the vicinity of inhomogeneously broadened absorption lines," Pure Appl. Opt. 5, 845-853 (1996).

38. J. Woollam, WVASE Manual: Guide to Using WVASE32 (WexTech Systems, Inc., 1996).

39. J. Sancho-Parramon, V. Janicki, and H. Zorc, "On the dielectric function tuning of random metal-dielectric nanocomposites for metamaterial applications," Opt. Express 18, 26915-26928 (2010). 